\title{
Identification of Sentinel Lymph Node in Breast Cancer with three Tracers (Radiocolloid, Methylene blue, and Indocyanine Green). (Case Report)
}

Borislav Kondov ${ }^{1}$, Viktor Gruev ${ }^{2,3}$, Sinisha Stojanovski ${ }^{4}$, Magdalena Bogdanovska-Todorovska ${ }^{5}$, Risto Colanceski ${ }^{1}$, Marija Srceva ${ }^{6}$, Anamarija Jovanovska ${ }^{4}$, Zlatko Stojkovski ${ }^{7}$, Goran Kondov $^{1 *}$

${ }^{1}$ University Clinic of Thoracic and Vascular Surgery, Medical Faculty, Skopje, Republic of Macedonia; ${ }^{2}$ Department of Electrical and Computer Engineering, University of Illinois at Urbana-Champaign, Urbana, IL, USA; ${ }^{3}$ Beckman Institute for Advanced Science and Technology, University of Illinois at Urbana-Champaign, Urbana, IL, USA; ${ }^{4}$ Institute of Pathophysiology and Nuclear Medicine, Faculty of Medical, Skopje, Republic of Macedonia; ${ }^{5}$ Institute of Pathology, Faculty of Medical, Skopje, Republic of Macedonia; ${ }^{6}$ University Clinic of Anesthesiology and Intensive Care, Medical Faculty, Skopje, Republic of Macedonia; ${ }^{7}$ City General Hospital "8 8 th of September," Skopje, Republic of Macedonia;

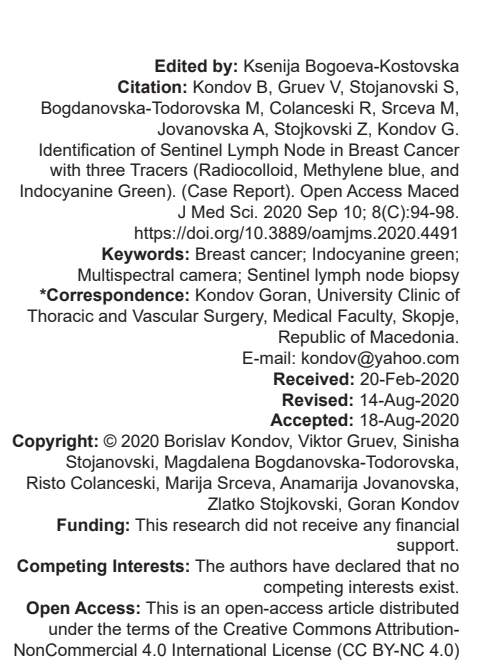

\section{Introduction}

The identification and biopsy of sentinel lymph node (SNL) in breast cancer for determination of axillary status and stage of the disease are already standard procedures in treatment of early-stage breast cancer [1], [2], [3].

The identification of the SNL is usually performed by radiocolloid injection that is further traced with a static gamma camera, i.e., intraoperatively with a hand-held gamma detector probe, as well as by injection of methylene blue.

Finding new agents that would identify the SNL, especially if they are not radioactive would be an important step in wider application of this method.

\section{Case Report}

In a 48-year-old patient with a malignant tumor in the upper-lateral quadrant of the left breast $13 \mathrm{~mm} \times 12 \mathrm{~mm}$ in size, with clinically node negative axilla, SNL was to be identified. This patient was involved in the clinical trial approved by the Institutional Review Board of the University of Illinois at UrbanaChampaign (IRB \#18947) and by the Agency for Drugs and Medical Instruments in Skopje, Republic of Macedonia (11-7442/3). The study complied with all ethical regulations. The study was registered on the website: clinicaltrials.gov (trial ID no. NCT03619967).

Four hours before surgery, a radionuclide $\mathrm{mTc}^{99}$-SENTI-SCINT (MEDI-RADIOPHARMA LTD. Hungary; human serum albumin nano sized colloid; 
$100-600 \mathrm{~nm})$ at a dose of $4 \mathrm{mC}(150 \mathrm{MBq})$ was injected at four points periareolar subcutaneously. Images with static gamma camera were made at 0 time, 60 min after application, and 90 min after application (Figure 1).

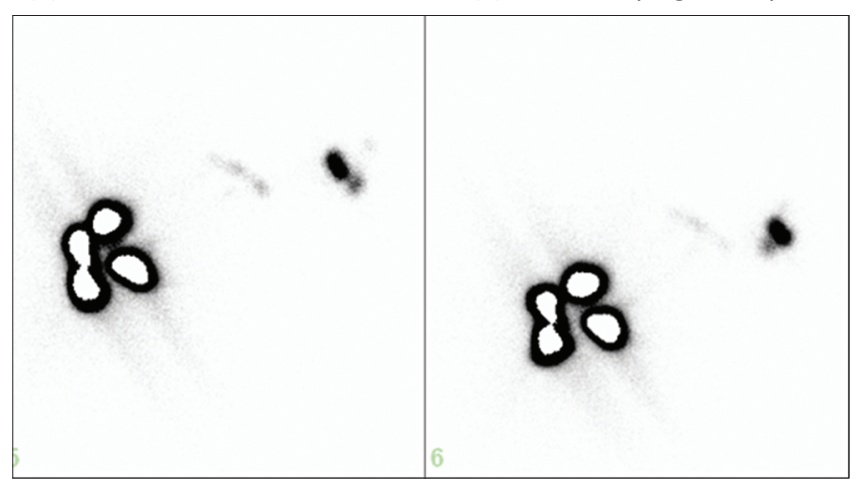

Figure 1: Static scan preoperatively 90 min after application

After detection of SNL with the static gamma camera, mapping of the SNL with radioactive tracer followed. At the same time, preoperatively, single-photon emission computed tomography scan was made that visualized the SNL and its relation with the surrounding structures (Figures 2 and 3 ).

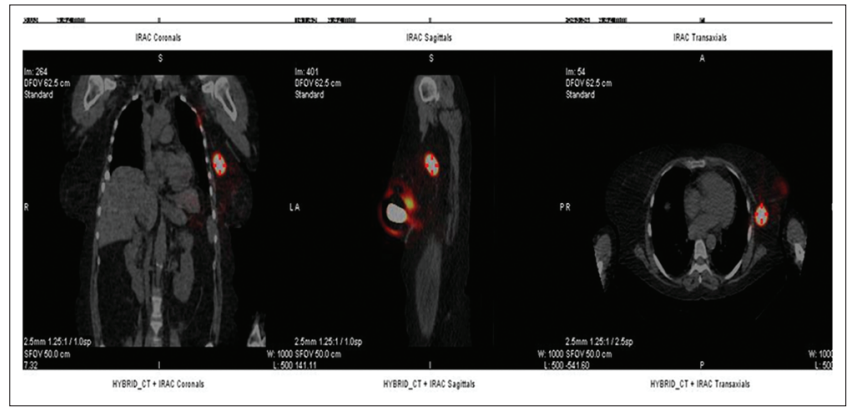

Figure 2: Single-photon emission computed tomography scan preoperatively

After mapping the SNL, the patient underwent a surgery under general anesthesia. This was followed by injection of $20 \mathrm{ml} 0.05 \%$ sterile solution of methylene blue at low-medial breast site in the direction towards the

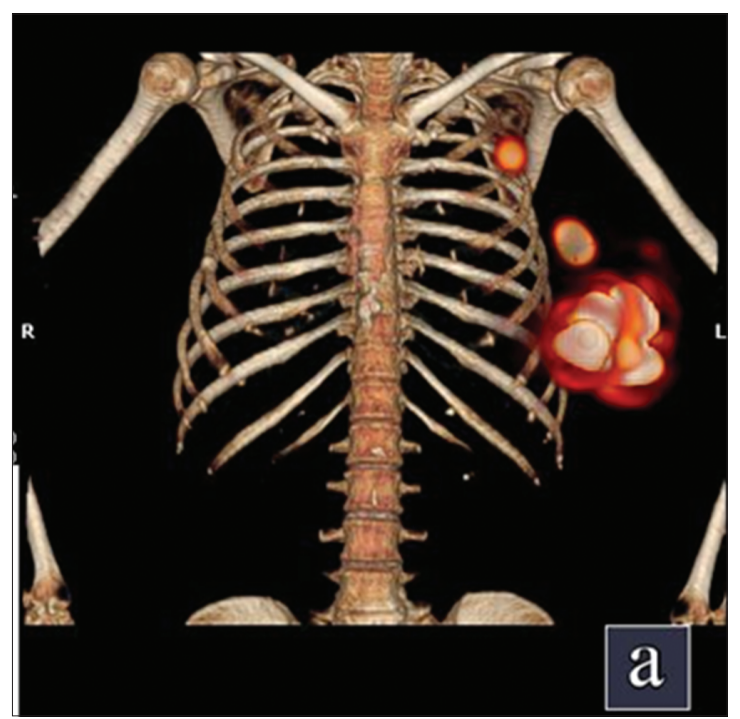

Figure 3: Single-photon emission computed tomography scan preoperatively axilla. After application of methylene blue, periareolar injection of $2 \mathrm{ml}$ (5 mg) indocyanine green (ICG) (ICG for injection, USP (Patheon Italia S.p.A.) at four sites followed. The breast was massaged for $10 \mathrm{~min}$ to distribute the dye toward the axillary lymph nodes. Both ICG and methylene blue were imaged around $30 \mathrm{~min}$ post-administration with a custom fluorescent camera.

Cleaning/cleansing of the surgical field and isolation was the next steps.

A small incision in the axilla $1-2 \mathrm{~cm}$ was made on a previously defined site for the identification of the SNL.

Tracing of the injected radiocolloid and dyes as well as identification of the SNL intraoperatively were performed with:

- Hand-held gamma detector probe EUROPROBE SYSTEM CE 0459

- $\quad$ Standard color camera

- Multispectral camera, which can detect nearinfrared fluorescence (NIR) that is emitted by ICG and methyline blue, respectively. The SLN mapping and resection were imaged with our bio-inspired sensor under simultaneous surgical light illumination (60 kLux) and two laser light excitations with $10 \mathrm{~mW} / \mathrm{cm}^{2}$ intensity at $780 \mathrm{~nm}$ and $665 \mathrm{~nm}$. The imaging and illumination systems were set up at a 1-m working distance. The image sensor was equipped with a Canon EF $50 \mathrm{~mm}$ f/1.2 lens that was focused on the patient and adjusted to an aperture of $f / 2.2$. The sensor was configured to provide a $0.1-\mathrm{ms}$ exposure to the color pixels to ensure non-saturated color images, and 33-ms exposure to the NIR pixels to ensure high SNR fluorescence signals and imaging rates of 30 FPS. ICG and MB fluorescence from resected lymph nodes was compared against visual detection and radioactive activity (Figure 4)

Macroscopic visualization of blue-colored tissues and lymph nodes.

Lymph nodes with blue coloration and radioactivity were excised and sent in frozen sections for histological analysis. The wound site was imaged with a custom bio-inspired camera capable of detecting fluorescence from ICG and MB. No clinical decision was made based on the fluorescence data because of the IRB protocol. The resected samples were compared for fluorescence, visual coloration, and radioactive activity.

After resection of the lesions, the axillary basin was locally examined for present radioactivity and coloration.

Three nodes with surrounding fat tissue were excised based on coloration and radioactive activity. A total of six nodes were detected in our patients. All of them were confirmed to be node negative (no metastatic deposits) on frozen sections as well as on paraffin sections. 
While waiting for the biopsy result (e.g., tempore analysis), a surgical procedure was undertaken for breast cancer treatment. Quadrantectomy of the upperlateral quadrant of the left breast was performed where the tumor was located. As soon as, the result from the axillary lymph nodes was obtained revealing that there were no metastatic deposits, the surgical wound was closed in layers.

It was confirmed in this patient that bluedye colored lymph nodes were also hot (filled with radioactivity), which was detected with a hand-held gamma detector probe, but they were also fluorescent (fluorescent-colored) detected with a multispectral camera. In fact, it was confirmed that ICG dye is suitable for identification/mapping of SNL in the armpit.

\section{Discussion}

The procedure for the identification of SNLs was described for the $1^{\text {st }}$ time in a parathyroid gland cancer study by Gould in 1960. Years later, in 1977 Cabanas presented evidence of the existence of SNLs in penile carcinoma. The Cabanas' hypothesis was that the diffusion of malignant cells in lymphatic organs follows an orderly, predictable pattern: It first affects one node, which he named the sentinel node, and then it is spread to the remaining lymph nodes of the drainage basin. By detecting and histological examination the SNL, the condition of the other lymph nodes of that basin can be predicted. This method was used for the first time for the clinical detection of breast cancer in 1992 by Krag, who had used radioactive technetium sulfur colloid for the mapping of the SNLs. Using this method, the SNL was detected in $83 \%$ of the cases. In 1994, for the $1^{\text {st }}$ time Armando Giuliano declared this method to be safe in the determination of axillary lymph node status and he used isosulfan vital blue dye to identify SNL in $89 \%$ of the cases. This procedure was later also used by Umberto Veronesi. Albertini used both of these methods and increased the identification rate to $92 \%$ [4], [5]. In 2005, Veronesi published his long-term initial results in the detection of SNLs; he presented the benefits of this method and in 2006 he suggested it for the determination of the disease stage [1], [2], [3].

Today, metadata for the detection and the biopsy of SNLs is a standard method in all protocols for the treatment of armpit in early-stage breast cancer with clinically negative axillary lymph nodes (there are no enlarged lymph nodes in the armpit clinically detected). If histological examination shows that the SNLs are not affected with metastatic deposit, then axillary lymphadenectomy is not performed, but in cases of metastatic deposits axillary lymphadenectomy have to be realized.
The need for performing metadata for the detection and the biopsy of SNLs is a result of the following facts:

- $\quad$ Recognition of the breast cancer biology helps in understanding that axillary lymphadenectomy has its role in the disease stage rather than in the therapeutic purposes

- In $40-60 \%$ of patients (depending on active mammography screening), there are no metastatic deposits in the armpit

- In cases of clinically negative armpit (enlarged lymph nodes in the armpit are not clinically detected), no metastatic changes are detected in $70 \%$ of those cases and no axillary lymphadenectomy is made.

Today, for the detection of the SNLs it is standard procedure to use radioisotope $\mathrm{mTc}^{99}$-SENTISCINT (MEDI-RADIOPHARMA LTD. Hungary; human serum albumin nano sized colloid; 100-600 nm) at a dose of $4 \mathrm{mCi}(150 \mathrm{MBq}$ ), divided into four separate doses (each dose at $1 \mathrm{mCi} / 37 \mathrm{MBq}$ ), applied at four sites periareolar subcutaneously, and isosulfan vital blue dye or methylene blue at our clinic.

Using this approach, the SNLs are detected in $98 \%$ of the cases. In conditions where after the radiocolloid injection the static scans performed before surgery prove the existence of the SNL, the accuracy reaches $100 \%$.

Radioisotope usage is related to radioactive radiation, requires licensing, has regulatory issues around handling and disposal of waste, necessary for the Institute for Nuclear Medicine, and may be logistically unavailable or difficult to implement in some centers or developing-countries. This has led to the development of alternative methods such as superparamagnetic iron oxide, fluorescence techniques using ICG or fluorescein, computed tomography lymphography, and contrastenhanced ultrasound scans using microbubbles. The newest techniques will potentially enable a more widespread adoption of this procedure; however, a common barrier for these techniques is the lack of standardization and no randomization of trials to evaluate their effectiveness against the current standard of care [6].

ICG dye is comprised small molecules of a fluorescent dye that can be detected with multispectral cameras that can detect light in the near-infrared spectrum. ICG is a cyanine dye used in medical diagnostics. It is used for determining cardiac output, hepatic function, liver and gastric blood flow, perfusion of lung, detecting SNLs, and for ophthalmic angiography. ICG binds tightly to plasma proteins and becomes confined to the vascular system. ICG has a half-life of 150-180 s and is removed from circulation exclusively by the liver to bile juice. The advantages of using this dye in the detection of SNLs are its non-toxic feature; it is applied locally at minimum doses; it moves quickly, and it is presented in the nodes/SNLs in an excellent 
manner. The frequencies of side effects were only $0.2 \%$. The camera can display movement of the dye under healthy skin without dying the tissue visibly. Due to its molecule's small size, it can quickly pass through the SNLs and present itself in the upper nodes too.

Guo et al. in their study comprising 68 patients proved that ICG was superior to blue dye for SNL identification. Using a photodynamic dye, movement of the dye through the lymphatic route could be observed in vivo. None of the patients experienced adverse effects associated with local application [6].

In 2016, Aydogan et al. presented their initial results of SNL biopsy under ICG guidance in four patients. They emphasized that ICG is a non-toxic substance, invisible under white light, has a short lifetime, but it is perfectly visible in the infrared spectrum that can be visualized with a multispectral camera. The disadvantage of this method is in the need of a nearinfrared camera that can capture fluorescence up to a depth of $21 \mathrm{~mm}$ from the skin surface. Furthermore, another limitation is the unfavorable influence of visualization by surgical lamps, and hence, very often it is necessary to use filters for the lamps or to use specially designed lamps with defined light spectrum [7].

Vidal-Sicart et al. in a study from 2013 discusses the identification of SNL indicating the use of a radiotracer and a vital blue dye as standard techniques. However, he has also pointed out ICG as a new fluorescence dye tracer that can be detected with specially designed cameras, which detect the near-infrared spectrum of beams. The added benefit would be the combined application of radiocolloid and a fluorescence dye as an integrated tracer that may be seen with two techniques for monitoring [8].

Takemoto et al. obtained better results in the detection of SNLs if ICG was combined with indigo carmine blue than if ICG was applied alone [9].

Pitsinis et al. have presented the advantages of using ICG in SNL detection compared to the injection of vital blue dye alone. They applied ICG in 50 patients and showed a detection rate of $100 \%$ in comparison with vital blue dye where it reached $96 \%$. To use ICG it is necessary to have a photodynamic eye, which registers fluorescence in near-infrared spectrum. These results are similar to those presented in the literature, where the accuracy of ICG in SNL detection is 99-100\% while for vital blue is $83-93 \%$. The added benefits of using ICG are in the fact that it gives almost no allergic reactions and does not visibly dye the tissue [10].

Zhang et al., in 2016, performed a meta-analysis to investigate the advantages of using ICG in the detection of SNLs. They performed a systematic literature search and found 67 published studies including 2595 patients. The selected studies conformed to all the criteria of randomization, additionally lymphadenectomies were made in only six studies after SNL identification. After analyzing and comparing these studies, an ICG method was found viable in the detection of SNLs. Furthermore, currently radiocolloid and vital blue dye are considered as standard methods of care in the detection of SNL.

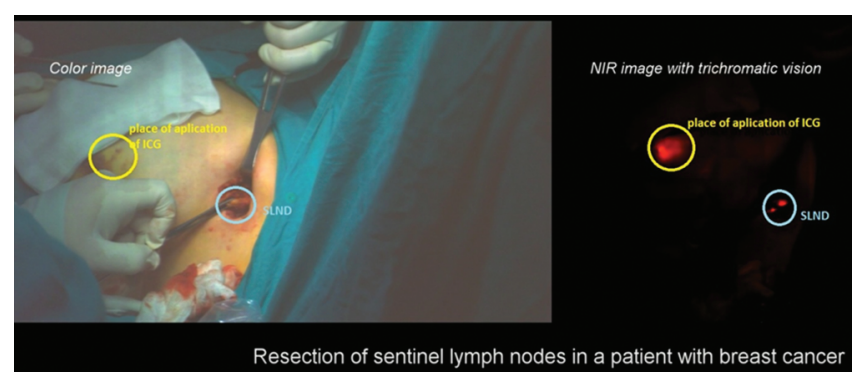

Figure 4: Comparison of the finding presented with color camera and multispectral camera after application of indocyanine green

\section{Conclusion}

The authors confirm ICG in the detection of SNL to have an accuracy of $98 \%$ with high sensitivity and specificity. Having in mind that isotopes are not used, the benefits of this method are even more important [11].

Our case report along with the studies from the literature lead to the conclusion that usage of ICG in the identification of SNLs is a method that is useful to be applied in practice, especially having in mind that it yields a high detection rate, high sensitivity, high specificity, has no adverse effects, and no radioactivity.

\section{References}

1. Veronesi U, Paganelli G, Viale G, Luini A, Zurrida S, Galimberti V, et al. Sentinel-lymph-node biopsy as a staging procedure in breast cancer: Update of a randomised controlled study. Lancet Oncol. 2006;7(12):983-90. https://doi.org/10.1016/ s1470-2045(06)70947-0

PMid:17138219

2. Zavagno G, De Salvo GL, Scalco G, Bozza F, Barutta L, De Bianco $\mathrm{P}$, et al. A randomized clinical trial on sentinel lymph node biopsy versus axillary lymph node dissection in breast cancer: Results of the sentinella/GIVOM trial. Ann Surg. 2008;247(2):20713. https://doi.org/10.1097/sla.0b013e31812e6a73 PMid:18216523

3. Gherghe M, Bordea C, Blidaru A. Sentinel lymph node biopsy (SLNB) vs. Axillary lymph node dissection (ALND) in the current surgical treatment of early stage breast cancer. J Med Life. 2015;8(2):176-80. https://doi.org/10.32388/eobekl PMid:25866575

4. Reintgen M, Kerivan L, Reintgen E, Swaninathan S, Reintgen D. Breast lymphatic mapping and sentinel lymph node biopsy: State of the art: 2015. Clin Breast Cancer. 2016;16(3):155-65. https://doi.org/10.1016/j.clbc.2016.02.014

PMid:26952594

5. Veronesi U, Galimberti V, Mariani L, Gatti G, Paganelli G, 
Viale G, et al. Sentinel node biopsy in breast cancer: Early results in 953 patients with negative sentinel node biopsy and no axillary dissection. Eur J Cancer. 2005;41(2):231-7. https:// doi.org/10.1016/j.ejca.2004.05.009

PMid:15661547

6. Guo W, Zhang Li, Ji J, Gao W, Liu J, Tong M. Breast cancer sentinel lymph node mapping using near-infrared guided indocyanine green in comparison with blue dye. Tumor Biol. 2014;35(4):3073-8. https://doi.org/10.1007/s13277-013-1399-2

7. Aydoğan F, Arıkan AE, Aytac E, Velidedeoğlu M, Yılmaz MH, Sager MS, et al. Sentinel lymph node biopsy under fluorescent indocyanin green guidance: Initial experience. Ulus Cerrahi Derg. 2016;32(1):50-3. https://doi.org/10.5152/ucd.2015.2832 PMid:26985159

8. Vidal-Sicart S, Giammarile F, Giuliano M, Olmos RA. Pre-and intra-operative imaging techniques for sentinel node localization in breast cancer. Imaging Med. 2013;5(3):275-91. https://doi. org/10.2217/iim.13.31
9. Takemoto N, Koyanagi A, Yasuda M, Yamamoto H. Comparison of the indocyanine green dye method versus the combined method of indigo carmine blue dye with indocyanine green fluorescence imaging for sentinel lymph node biopsy in breast conservative therapy for stage IIIA breast cancer. BMC Womens Health. 2018;18(1):151. https://doi.org/10.1186/ s12905-018-0646-5

PMid:30227837

10. Pitsinis V, Provenzano E, Kaklamanis L, Wishart GC Benson JR. Indocyanine green fluorescence mapping for sentinel lymph node biopsy in early breast cancer. Surg Oncol. 2015;24(4):375-9. https://doi.org/10.1016/j.suronc.2015.10.002 PMid:26555151

11. Zhang X, Li Y, Zhou Y, Mao F, Lin Y, Guan J, et al. Diagnostic performance of indocyanine green-guided sentinel lymph node biopsy in breast cancer: A meta-analysis. PLoS One. 2016;11(6):e0155597. https://doi.org/10.1371/journal. pone. 0155597

PMid:27280407 